OPEN ACCESS

Edited by:

Raya Khanin,

Memorial Sloan Kettering

Cancer Center, United States

Reviewed by:

Ignacio Maeso,

Centro Andaluz de Biología del

Desarrollo (CABD), Spain

Ildiko M. L. Somorjai,

University of St Andrews,

United Kingdom

Jordi Garcia-Fernandez,

University of Barcelona, Spain

"Correspondence:

Qi-Lin Zhang

zhangqilin88888@126.com

Lin-Feng Yang

yanglinfeng@bgi.com

Yu-Jun Wang

wangxiaochen528@hotmail.com

tThese authors have contributed equally to this work

Specialty section:

This article was submitted to Bioinformatics and

Computational Biology,

a section of the journal

Frontiers in Genetics

Received: 02 March 2019

Accepted: 21 August 2019 Published: 26 September 2019

Citation:

Zhang Q-L, Wang H, Zhu Q-H, Wang X-X, Li Y-M, Chen J-Y,

Morikawa $H$, Yang L-F and

Wang Y-J (2019) Genome-Wide Identification and Transcriptomic

Analysis of MicroRNAs Across

Various Amphioxus Organs Using

Deep Sequencing.

Front. Genet. 10:877.

doi: 10.3389/fgene.2019.00877

\section{Genome-Wide Identification and Transcriptomic Analysis of MicroRNAs Across Various Amphioxus Organs Using Deep Sequencing}

\author{
Qi-Lin Zhang ${ }^{1,2+*}$, Hong Wang ${ }^{1 \dagger}$, Qian-Hua Zhu ${ }^{3+}$, Xiao-Xue Wang ${ }^{1 \dagger}$, Yi-Min Li ${ }^{1}$, \\ Jun-Yuan Chen ${ }^{4}$, Hideaki Morikawa ${ }^{5}$, Lin-Feng Yang ${ }^{3 *}$ and Yu-Jun Wang ${ }^{1 *}$
}

${ }^{1}$ Guangxi Key Laboratory of Beibu Gulf Marine Biodiversity Conservation, Ocean College, Beibu Gulf University, Qinzhou, China, ${ }^{2}$ Faculty of Life Science and Technology, Kunming University of Science and Technology, Kunming, China, ${ }^{3}$ BGl Genomics, BGlShenzhen, Shenzhen, China, ${ }^{4}$ Evo-devo Institute, School of Life Sciences, Naniing University, Naniing, China, ${ }^{5}$ Faculty of Textile Science and Technology, Shinshu University, Ueda, Nagano, Japan

Amphioxus is the closest living invertebrate proxy of the vertebrate ancestor. Systematic gene identification and expression profile analysis of amphioxus organs are thus important for clarifying the molecular mechanisms of organ function formation and further understanding the evolutionary origin of organs and genes in vertebrates. The precise regulation of microRNAs (miRNAs) is crucial for the functional specification and differentiation of organs. In particular, those miRNAs that are expressed specifically in organs (OSMs) play key roles in organ identity, differentiation, and function. In this study, the genome-wide miRNA transcriptome was analyzed in eight organs of adult amphioxus Branchiostoma belcheri using deep sequencing. A total of 167 known miRNAs and 23 novel miRNAs (named novel_mir), including 139 conserved miRNAs, were discovered, and 79 of these were identified as OSMs. Additionally, analyses of the expression patterns of eight randomly selected known miRNAs demonstrated the accuracy of the miRNA deep sequencing that was used in this study. Furthermore, potentially OSM-regulated genes were predicted for each organ type. Functional enrichment of these predicted targets, as well as further functional analyses of known OSMs, was conducted. We found that the OSMs were potentially to be involved in organ-specific functions, such as epidermis development, gonad development, muscle cell development, proteolysis, lipid metabolism, and generation of neurons. Moreover, OSMs with non-organ-specific functions were detected and primarily include those related to innate immunity and response to stimuli. These findings provide insights into the regulatory roles of OSMs in various amphioxus organs.

Keywords: Branchiostoma belcheri, organ transcriptomics, microRNAs, deep sequencing, qRT-PCR 


\section{INTRODUCTION}

The phylum Chordata contains three subphyla members, including the Cephalochordata (e.g., amphioxus), Urochorda (e.g., ciona), and Vertebrata (Satoh et al., 2014). The origin and evolution of organs and tissues in chordates are two of the most important scientific questions in evolutionary developmental biology (evo-devo) (Holland and Chen, 2001). However, with the exception of a recent report by Marlétaz et al. (2018), genetic information from different organs of amphioxus as the sister lineage to all other chordates remains largely unstudied, which limits our understanding for molecular mechanisms of organ function formation in chordate as a vertebrate outgroup and origin of organ complexity in vertebrates. Amphioxus, also known as lancelets, which belong to the subphylum Cephalochordate, retains some of the structural morphology characteristics observed in common ancestors between cephalochordates and vertebrates from the Cambrian period of $\sim 530$ million years ago (Chen et al., 1999; Putnam et al., 2008). Therefore, cephalochordates are key experimental animals for the study of evo-devo, evolutionary origins of organs, and comparative immunology of vertebrates (Putnam et al., 2008; Huang et al., 2014).

Noncoding RNAs (ncRNAs) regulate many biological processes, such as development, apoptosis, metabolism, differentiation, and the function formation of cells in animals (Arner and Kulyté, 2015). Of the ncRNAs, those known as microRNA (miRNA, 22 nucleotides) are the most extensively investigated types. In general, many of these miRNAs are transcribed from introns of protein-coding genes and negatively regulate expression of their targets by complementary base pairing to regions in the $3^{\prime}$ UTR (untranslated region) of mRNAs in animals (Campo-Paysaa et al., 2011). Furthermore, miRNAs may contribute to the function formation of different organs through their specific expression in amphioxus. It has been reported that miR-92 is specifically expressed in the hepatic cecum, gill, and intestines in amphioxus. It is believed to regulate the complement pathway by targeting $\mathrm{C} 3$ to promote the immune response against bacterial infection (Yang et al., 2013). Muscle-specific expression of miR-1 and miR-133 was clearly demonstrated using in situ hybridization using in amphioxus early larvae (Campo-Paysaa et al., 2011). Candiani et al. (2011) identified six miRNAs with specific expression in the nervous system of amphioxus by wholemount in situ hybridization. Both differentiating and mature neurons exhibited specific expression of miR-124. Thus, the body of literature suggests that miRNAs may be key regulatory molecules for the regional and specific function formation of different organs in amphioxus. So far, only miRNA expression profiles in three digestive and immune-related organs, gill, intestine, and hepatic cecum, have been obtained using microarray technology based on nucleic acid hybridization (Liao et al., 2017a). However, only three organ types were used, and microarray based on nonsequencing technology is not feasible for the identification of novel molecules in different organs (Gao et al., 2011), possibly hindering a systematic and accurate discovery and function analysis of organ-specific expressed genes.

Next-generation sequencing is a useful tool for the analysis of the expression profile of miRNAs. Its advantages are unbiased large-scale detection of small RNAs (sRNAs) at a genome-wide level, even for transcripts with low expression levels, and its ability to identify novel RNA miRNAs (Yao et al., 2012). To date, there has not been a comparative analysis of miRNA expression profiles among different organs in amphioxus using deep sequencing. In this study, deep sequencing was used to systematically analyze miRNAs purified from eight organs of adult Chinese amphioxus (Branchiostoma belcheri). In addition to demonstrate organspecific expression of a large group of known miRNAs and novel miRNA candidates among different organs, the corresponding target genes of these miRNAs were predicted, and the function of known organ-specific expressed miRNAs (OSMs) was explored. Then, the function of all OSMs was analyzed by enrichment of Gene Ontology (GO) terms using bioinformatics. The dataset obtained here will be a valuable resource for uncovering the potential roles of miRNAs in organ differentiation of amphioxus.

\section{MATERIALS AND METHODS}

\section{Ethics Statement}

This study was carried out in accordance with the recommendations of the Guide for the Care and Use of Laboratory Invertebrate Animals. The protocol was approved by the Ethical Committee of Researches of the Nanjing University.

\section{Sample Preparation}

Healthy adult individuals of $B$. belcheri were obtained from the Evo-Devo Institute of Nanjing University at Beihai City, Guangxi Province, China. Experimental animals were kept in acrylic tanks with filtered seawater, following our previously used methods (Liao et al., 2017b). These individuals were maintained for several days to empty their intestinal and hepatic cecum contents. Subsequently, each of the approximately 40 individuals was placed on ice and dissected to collect the eight specific organs (i.e., nerve cord, notochord, skin, intestine, hepatic cecum, muscle, gill, and ovary) used in this study. Each organ type obtained from different individuals was pooled together in a $1.5-\mathrm{mL}$ RNasefree microcentrifuge tube and immediately frozen using liquid nitrogen and stored at $-80^{\circ} \mathrm{C}$. All experimental samples were stored at Beihai Marine Station of Nanjing University in Beihai, Guangxi Province, China.

Paraffin section of $B$. belcheri was generated according to previous methods (Yang et al., 2013). In brief, adult B. belcheri was fixed in $4 \%$ paraformaldehyde at $4^{\circ} \mathrm{C}$ for $12 \mathrm{~h}$, dehydrated with graduated ethanol, and embedded in paraffin. Then, the embedded amphioxus was cut into $10-\mu \mathrm{m}$ sections. After deparaffinization, organ sections were dyed using a hematoxylin and eosin staining kit (Yeasen, USA) following the manufacturer's manual. Next, the dyed sections were photographed by Olympus microscope DP71 (Olympus, Japan).

\section{RNA Extraction, Library Construction, and Sequencing}

The total RNA of each organs type was extracted using Trizol reagent (Invitrogen, USA), according to the manufacturer's 
protocol. Residual DNA contamination was removed by RNase-free DNase (Qiagen, Germany). RNA concentration and quality were initially assessed using a BioPhotometer Plus (Eppendorf, Germany). Next, an Agilent 2100 Bioanalyzer (Agilent Technologies, USA) was employed to further verify RNA structural integrity and quality. RNA samples with an RNA integrity number (RIN value) $\geq 7$ were used in further experiments. Preparation of small-RNA libraries was performed using Illumina TruSeq Small RNA Library Preparation Kits (Illumina, USA). Quality of the small-RNA library was assessed using the Agilent 2100 Bioanalyzer (Agilent Technologies) and an ABI StepOnePlus Real-Time PCR System (Applied Biosystems, USA). Deep sequencing of the eight prepared libraries was performed on an Illumina HiSeq4000 platform (Illumina) with 50-bp single-end reads at the Beijing Genomics Institute (BGIShenzhen, China).

\section{Analysis of Deep Sequencing Data}

Clean reads were obtained from the raw data by removing lowquality tags, reads with poly-N tails or 5' adapter contaminants, reads with $3^{\prime}$ null adapters, and insert null tags. Next, clean sRNA tags were mapped to the B. belcheri genome (v18h27.r3; http://genome. bucm.edu.cn/lancelet/, early available) using Bowtie2 (parameter: -q -L 16 -phred64 -p 6) to analyze their expression and distribution across the reference genome (Langmead et al., 2009). To discard tags originating from rRNAs, tRNAs, snRNAs, snoRNAs, and sRNA tags, a BLAST search was conducted against the Rfam 11.0 and the NCBI GenBank databases. RepeatMasker software (http:// www.repeatmasker.org/) and genome-based mapping information were used to remove tags from repeat regions and protein-coding sequences (Zhong et al., 2015). The sRNA sequences were identified by performing a BLAST search in miRBase21.0 (Griffiths-Jones, 2006), allowing a maximum of two mismatches to known miRNA.

\section{Identification of Novel miRNAs and Homologous miRNAs}

The remaining sRNA tags originating from exon sense and intron and intergenic regions were used to identify novel miRNAs and their precursors. The prediction software mirDeep2 was used to identify novel miRNAs using default options recommended in the software manual (Friedländer et al., 2012). MirDeep2 has a substantially improved algorithm for the identification of miRNAs from RNA sequencing data of animal groups and can predict canonical and noncanonical miRNAs with an accuracy of $98.6 \%$ to 99.9\% (Friedländer et al., 2008; Friedländer et al., 2012). To ensure the accurate discovery of novel miRNAs through verification of the secondary structures, novel miRNA precursors (pre-miRNAs) were analyzed using RNAfold (Höner zu Siederdissen et al., 2011) to estimate whether the secondary structure of the precursor is a perfect stem-loop formation. Furthermore, the predicted novel miRNA tags/sequences with mapped tags of fewer than five were discarded (Zhang et al., 2017a), and the remainder retained as the novel mirDeep2 miRNAs. Meanwhile, the additional prediction software Mireap (Yuan et al., 2013) was used to discover novel miRNAs by predicting the hairpin structure, dicer cleavage site, and minimum free energy of the unannotated sRNA tags that had been mapped to the genome. Those tags with a minimum free energy value of the folding precursor $-19 \mathrm{kcal} / \mathrm{mol}$ or less were kept as novel miRNAs identified by Mireap. The intersection of identified miRNAs by mirDeep 2 and Mireap was retained. Then, these identified miRNAs were further filtered as the final novel miRNA set under strict criteria (Huang et al., 2017): 1) the presence of both $5 p$ and $3 p$ strands in the read dataset and 2) their overlap with a $\sim 2$ nt overhang on each side when the hairpin is folded. Subsequently, novel miRNAs and B. belcheri known miRNAs above annotated were searched in miRBase21.0 animal database (excluding $B$. belcheri dataset), allowing for a maximum of two mismatches, and those that were annotated as known miRNAs of other animals were considered to be conserved/homologous.

\section{Normalization of miRNAs Expression and Identification of Organ-Specific miRNAs}

Expression levels of all miRNAs (conserved and novel miRNAs) were normalized using transcripts per million (TPM) values (Zhong et al., 2015). To estimate the organ specificity of a miRNA, an entropy-based metric that relies on Jensen-Shannon (JS) divergence was employed to calculate specificity scores (ranging from 0 to 1 ) based on previous descriptions of the JS algorithm (Cabili et al., 2011). This specificity metric quantifies the similarity between a transcript's expression pattern across tissues and another predefined pattern that represents the extreme case in which a transcript is expressed in only one tissue. Therefore, a perfect organ-specific expression pattern is scored as $\mathrm{JS}=1$, indicating that a transcript is expressed in only one organ type (Cabili et al., 2011). Specifically, index $\tau$ was calculated according to previously published methods using a custom Perl script to detect the organ specificity of miRNA expression (Yanai et al., 2005). For a given transcript, the index $\tau$ is defined as $\tau=\sum_{i=1}^{N}\left(1-x_{i}\right) / N-1$, where $N$ is the number of organs, and $x_{i}$ is the expression value in the $i$ th organ normalized by the maximal expression value across all of the organs ( $\mathrm{Li}$ et al., 2015). First, it was determined whether expression was organ-specific for each miRNA. If miRNAs were observed to have values for $\tau>0.85$, they were considered to be expressed in an organ-specific manner (OSMs) (Cabili et al., 2011). Next, if OSMs were observed, JS scores were used to evaluate organs for the specific expression for each OSM as previously described (Cabili et al., 2011; Li et al., 2015). Finally, each $\tau$ value was compared with the other JS scores (corresponding to each of the eight organs) for each miRNA. Organ specificity of miRNA expression and the respective expression-specific organs were successively confirmed.

\section{Determination and Functional Analysis of Potential miRNA Targets}

The potential target genes of OSMs were predicted using two bioinformatics tools. The first was RNAhybrid (Kruger and Rehmsmeier, 2006), and the default parameters were used as follows: -b 100 -c -f 2,8 -m 100000 -v 3 -u 3 -e -20 -p 1 -s 3utr_Bb. The second tool used was miRanda (Betel et al., 2008), with the 
following default parameters of -en -20 -strict being used. The intersection point for the results from two prediction tools was assumed to be the set of reliably predicted target genes of the OSMs. These potential targets were selected for downstream analyses. To determine the functions of the predicted OSMregulated target genes, the genes that were predicted target genes of all miRNAs identified in this study were annotated using a basic local BlastX tool (Kent, 2002), using the default parameters in the NCBI nonredundant (NR) animal database. To further understand the regulatory function of OSMs in different organs of B. belcheri, known OSM (analyzed based on B. belcheri miRNAs previously identified and stored at miRBase database) target genes predicted by bioinformatics were filtered and extracted according to the following criteria: 1) target genes with unknown annotation and hypothetical proteins were removed, and 2) multiple targets with the same gene description were filtered, with only one being retained as the representative. Next, GO annotation of each gene was extracted using Blast2GO pipeline (Conesa et al., 2005) according to the NR annotation. Because annotation of model animals is full, while that of nonmodel species is lacking, thus sequences of $B$. belcheri were searched in libraries of all the animal species in the GO term analysis to obtain adequately best match. We performed the GO enrichment analysis for the predicted target genes of the OSMs using Fisher exact test in Blast2GO pipeline (Conesa et al., 2005), and outputs ( $P$ values) of the software were used to perform multiple test corrections by false discovery rate (FDR). Gene Ontology terms presenting FDR of less than 0.05 were retained. The redundant GO terms were then reduced using the GO trimming tool (Koop et al., 2011). Gene Ontology terms for biological processes were retained to represent the enriched functions of predicted target gene clusters.

\section{Quantitative Real-Time Polymerase Chain Reaction}

Collection of the eight organs and extraction of their total RNA, including sRNAs, were performed according to the aforementioned methods. For each organ type, three biological replications were used. Mature miRNA expression was measured using Taqman probe kits (Applied Biosystems) customized for each gene, including the RT primers, polymerase chain reaction (PCR) primers, and Taqman probes. The product information for each miRNA probe is presented in Supplementary File 1. We selected $\mathrm{U} 6$ as a housekeeping gene. Each reaction was conducted in three technical replicates. Relative expression levels of target miRNAs were normalized using the $2^{-\Delta \Delta \mathrm{Ct}}$ method (Livak and Schmittgen, 2001). The data shown are presented as the mean $\pm \mathrm{SD}$, and figures were generated by SigmaPlot 12.0 (Systat Software Inc., San Jose, CA). According to methods described in our previous studies (Zhang et al., 2017b), consistency of miRNA expression patterns between deep sequencing and quantitative real-time PCR (qRT-PCR) was evaluated by calculating the Pearson correlation coefficient and its significance ( $P$ value) level in IBM SPSS statistics 22 software (International Business Machines Corp., New York, USA).

\section{RESULTS}

\section{Overall Assessment of the miRNAome of Different Organs}

Approximately 34 million clean reads for each of the eight organ samples, including skin, ovary, notochord, nerve cord, muscle, intestine, hepatic cecum, and gill, were obtained after filtering lowquality reads and contaminants (Figures 1A-C and Supplementary File 2). All of the organ samples showed peak length of the miRNAs

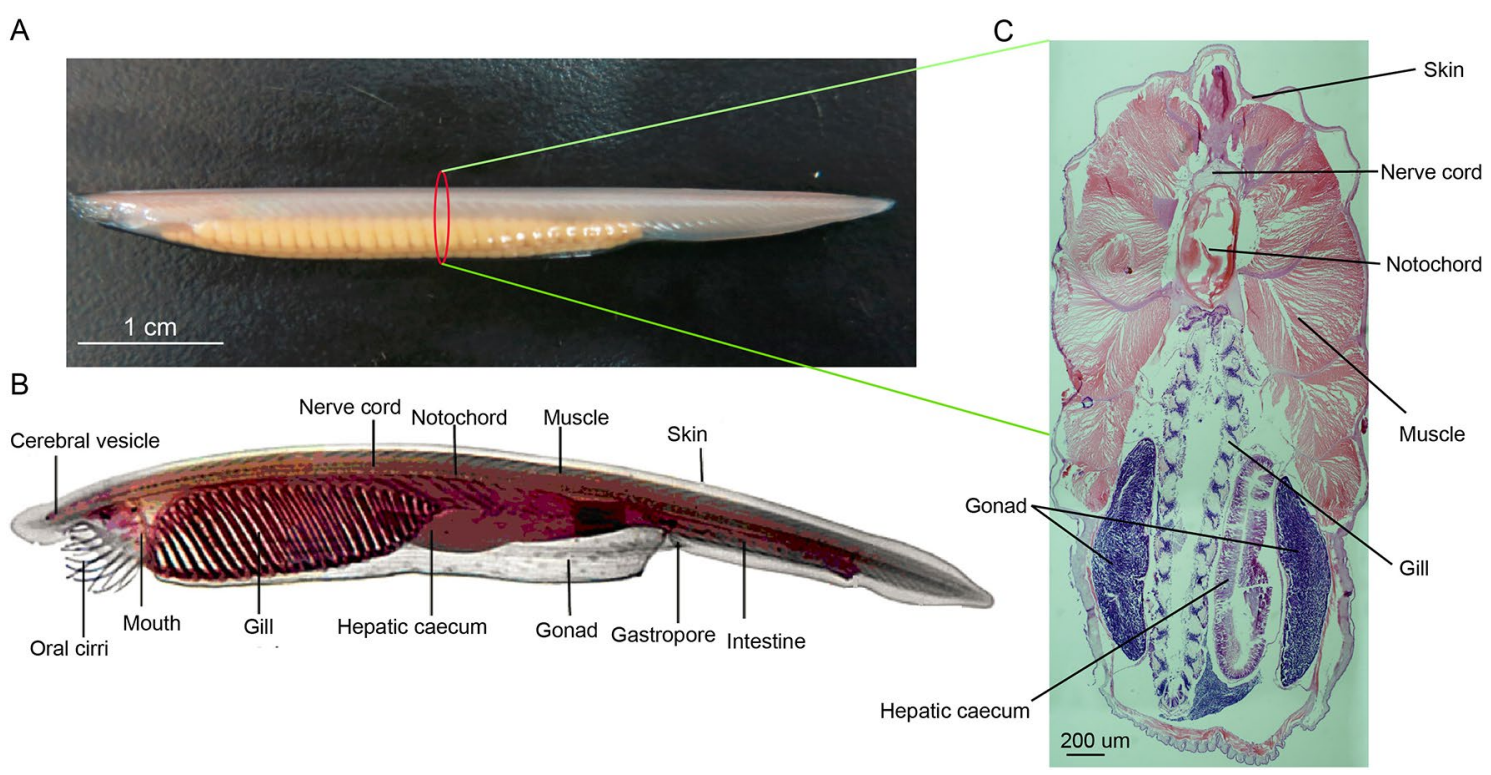

FIGURE 1 | (A) Female adult amphioxus Branchiostoma belcheri (photographed by EOS 6D digital single-lens reflex cameras (Canon, Japan)). (B) An overview of body structure of adult B. belcheri (adapted from http://faculty.baruch.cuny.edu/jwahlert/bio1003/chordata.html). (C) Hematoxylin and eosin (H\&E) staining on transverse sections of an adult Branchiostoma belcheri, with staining of nucleus purple and the cytoplasm pink. 
at about 22 to $23 \mathrm{nt}$ (Supplementary File 3), as has been previously described for B. belcheri and other animals (Zhang et al., 2017a). All sequencing data have been released to the NCBI SRA database under accession number SRR9951226-SRR9951233. The ratios of total clean tags that mapped to the $B$. belcheri genome ranged from $74.61 \%$ to $82.23 \%$, indicating that a set of reliable clean tags was obtained (Zhang et al., 2017a). To minimize false-positive signals, only tags with TPM values 5 or greater were retained for further bioinformatics analysis. Based on the annotated results of sRNA, it is found that miRNAs occupied a high proportion of sRNA tags in B. belcheri organs investigated in this study ( $>50 \%$, Supplementary File 4), indicating that miRNA molecules were adequately enriched.

\section{Identification of Known, Conserved, and Potential Novel miRNAs in $B$. belcheri}

A total of 167 known miRNAs, distributed among 82 miRNA families, were expressed in at least one organ type (Table 1). In miRBase21.0, 173 mature bbe-miRNA sequences have been stored, and approximately $97 \%$ of these were identified in the current study. Of these previously annotated miRNAs, approximately $93 \%$ are expressed in the nerve cord. Moreover, 138 known miRNAs were identified to be homologous with those of Branchiostoma floridae, 39 of which are homologous to miRNAs in vertebrates (Supplementary File 5). Together, this indicates that these 39 vertebrate miRNAs have evolved in ancient chordates. However, and 99 of the known miRNAs have only been detected in amphioxus.

A total of 23 potential novel miRNAs were shared (expressed in all eight organs) (Supplementary File 6) across all organs sequenced in this study. These were analyzed further for the detection of the organ expression specificity and specifically expressed organs by calculating their respective $\tau$ and then JS scores, respectively. Predicted precursor structure of these novel miRNAs is presented in Supplementary File 7. Furthermore, novel_mir1 was detected and is conserved with bfl-miR-4871-3p of B. floridae (Jin et al., 2017; Zhang et al., 2017a). Homologs of the remaining novel miRNAs were not detected, indicating that these miRNAs may potentially be lineage-specific in B. belcheri. Alternatively, these remaining novel miRNAs may be existing in other species but have not been detected yet.

\section{Identification and Functional Enrichment Analysis of OSMs}

Of all $B$. belcheri miRNAs identified here, 79 OSMs $(41.58 \%)$ showed organ-specific expression $(\tau>0.85)$ (Supplementary File 8).

TABLE 1 | Statistics summary of miRNA in eight organs of Branchiostoma belcheri.

\begin{tabular}{lcc}
\hline Organ types & Known miRNAs & Novel miRNAs \\
\hline Nerve cord & 160 & 10 \\
Notochord & 147 & 8 \\
Skin & 154 & 8 \\
Intestine & 151 & 8 \\
Hepatic cecum & 152 & 12 \\
Muscle & 157 & 12 \\
Gill & 155 & 13 \\
Ovary & 138 & 7 \\
Total amount & 167 & 23
\end{tabular}

Furthermore, the distribution tendency of maximal JS scores is presented in Figure 2. Among them, 68 OSMs were observed to be conserved with those of $B$. floridae as well as other vertebrates, accounting for $86.08 \%$ of all OSMs (Supplementary File 8). Among the eight organs, the nerve cord sample showed the highest number of OSMs (26), followed by the gill (20), hepatic cecum (11), muscle (8), and the skin (7). The ovary (3), notochord (2) and the intestine (2) had the lowest numbers of OSMs (Figure 3A). All OSMs are listed in Supplementary File 8, including known and novel OSMs. The known OSMs are also presented in Figure 3B.

The comprehensive miRNA catalog allows us to explore the potential functions of the miRNAs observed in B. belcheri. Here, 22,117 potential targeted genes were identified using both the RNAhybrid and miRanda software programs (Figure 3C). Using GO enrichment analysis, the putative target genes of the OSMs in each organ were to be enriched using the GO terms belonging to the "Biological Process" subcategory (Supplementary File 9). In the skin, 12 enriched GO terms were primarily related to response to stimulus and epidermis development. These terms also included "apoptotic process," "response to UV-B," "response to mechanical stimulus," and "epidermis development." For the ovary, the five GO terms obtained were overrepresented and associated with signaling processes, immune response and ovary development, which included "signaling," "defense response," and "gonad development. Unsurprisingly, GO terms enriched from predicted target genes of muscle OSMs were primarily involved in muscle development and morphogenesis. The intestine, hepatic cecum, and gill are considered to be digestive and immune tissues of amphioxus (Liu et al., 2009; Han et al., 2010). In these three organs, many GO terms involving immune and stimulus response, apoptosis, immune system, and metabolic and catabolic processes were widely enriched. Notably, the GO term "proteolysis" was specifically exhibited in the intestine. Meanwhile, it was observed

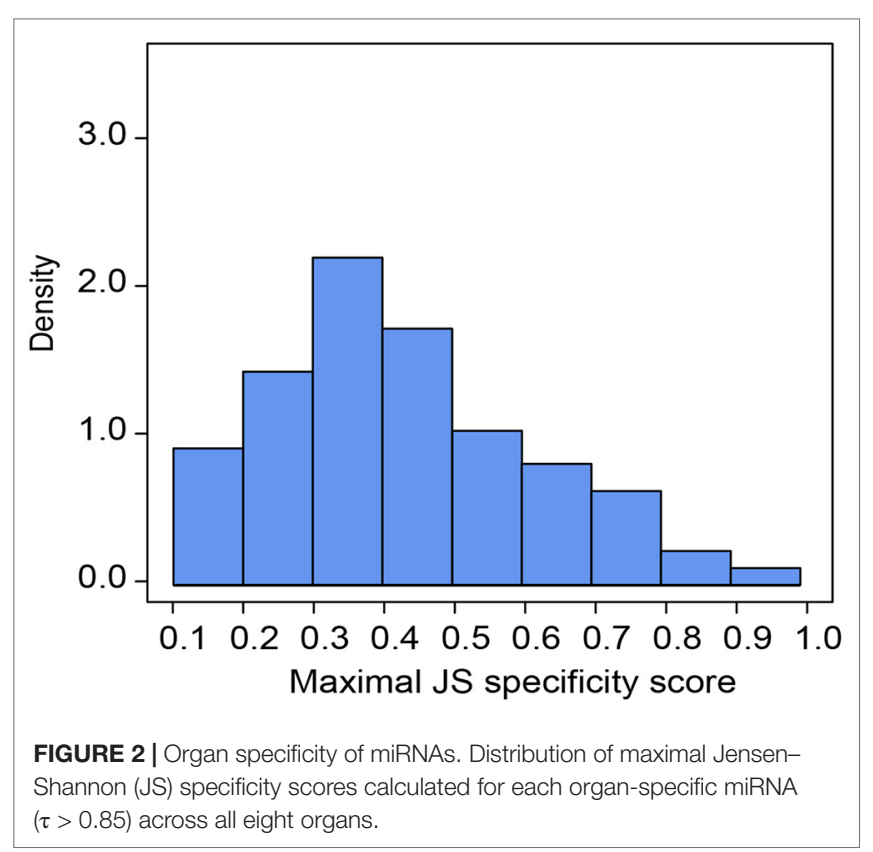


that OSMs of the hepatic cecum also specifically regulated target genes related to stimulus and organic metabolism. In the nerve cord, OSMs were primarily associated with stimulus and drug response, circadian rhythm, sensory behavior, and neural development and function. The GO terms enriched by predicted targets of notochord OSMs were related to cell process and function, phosphorylation, and development process.

\section{Analysis of Predicted Target Genes of Known OSMs}

Known OSM target genes predicted by bioinformatics were listed (Supplementary File 10). We found that some typical genes involved in stimulus response could be regulated by specific expression of several known OSMs in the skin, such as heat shock protein 70 (HSP70) and genes encoding nod-like receptors (NLR protein), mitogen-activated protein kinase 3
(MAP4K3), mucin-2-like (MUC2L) and tumor necrosis factor receptor superfamily member 11A (TNFRSF11A), Toll-like receptor 4 (TLR4), and collagen alpha-1 (XII) chain (COL12A1). Additionally, genes related to calmodulin regulation were found as predicted targets of skin OSMs, including calmodulin-related protein 3 (CALREP3), calcium-activated potassium channel subunit alpha-1 (KCNMA1), and calcium/calmodulin-dependent protein kinase type II subunit gamma (CAMK2G).

In the ovary, bbe-miR-2068-5p was predicted to regulate lipid metabolism function, reactive oxygen species, and gene encoding piwi-like 1 protein (PIWIL1), while bbe-miR-4873-5p participated probably in the regulation of genes involving stimulus and immune responses, such as mitogen-activated protein kinase kinase kinase15 (MAP3K15), WD repeat-containing protein 26 (WDR26), and ubiquitin-like modifier-activating enzyme 6 (UBA6).

In the notochord, it is found that genes potentially related to notochord development were regulated by known OSMs,
A

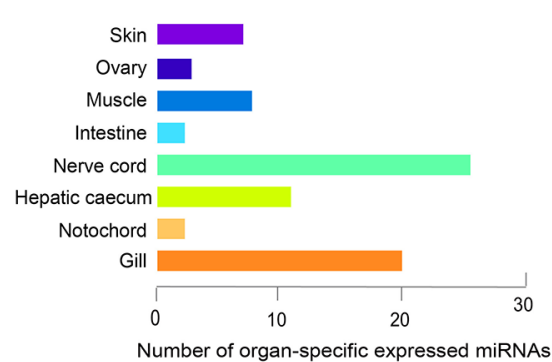

C

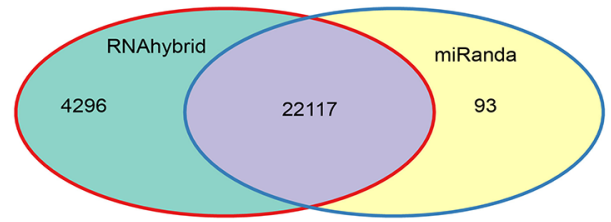

B

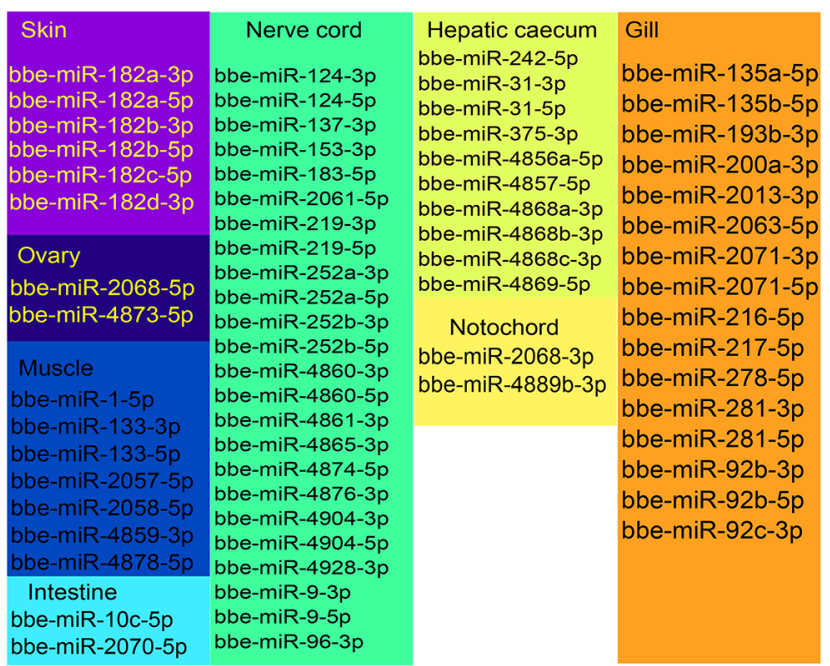

D

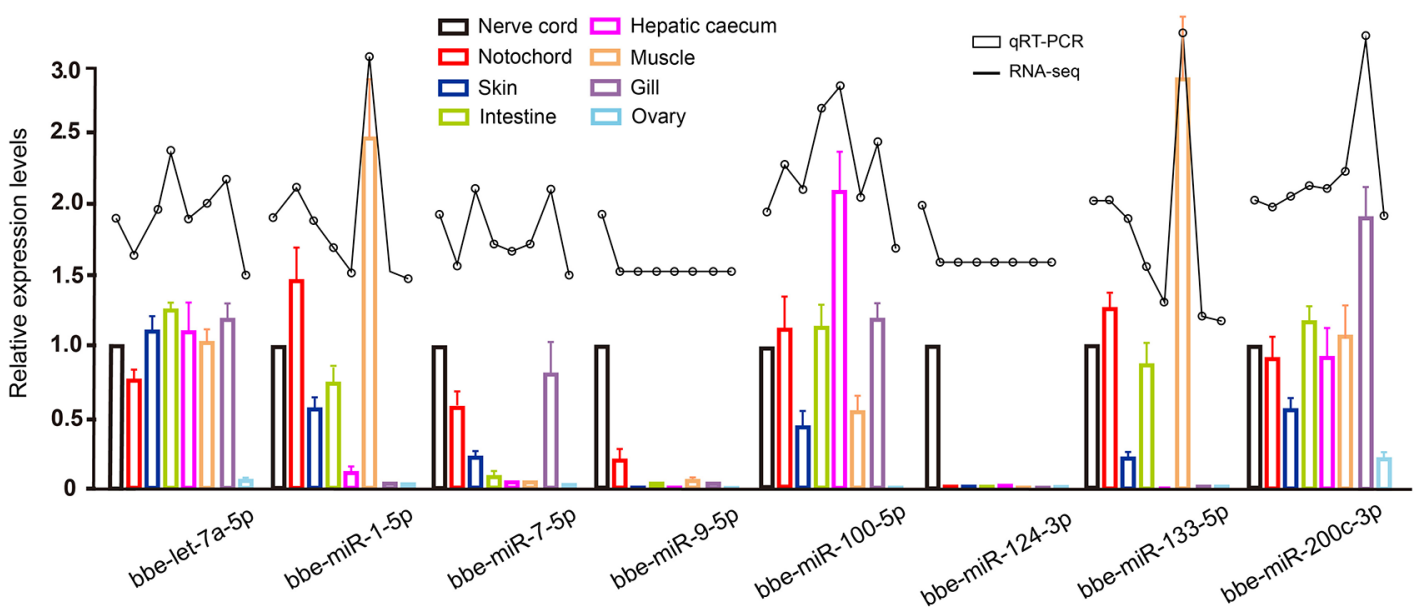

FIGURE 3 | (A) Number distribution of organ-specific miRNAs across eight organs. (B) The list of known organ-specific miRNAs in eight organs. (C) Number of predicted target genes of organ-specific miRNA using RNAhybrid and miRanda software. (D) Validation of expression of miRNAs by qRT-PCR experiment. Expression of eight selected randomly miRNAs was detected by quantitative real-time polymerase chain reaction in $B$. belcheri organs including the skin, ovary, muscle, intestine, hepatic cecum, gill, nerve cord, and notochord. 
including insulin-like growth factor 1 (IGF1), bone morphogenetic protein 3 (BMP3), notochord homeobox-like protein, muscle, and skeletal receptor tyrosine-protein kinase (MUSK).

In the nerve cord, various innate immune-related genes were found to be putative targets of OSMs, including complement (C1q1, complement factor $\mathrm{H}[\mathrm{CFH}]$, complement component factor $\mathrm{B} / \mathrm{C} 2[\mathrm{~B} / \mathrm{C} 2]$ ), pattern recognition receptors (PRRs) (NLR protein, RIG-I-like receptor LGP2), cytokines (interferon regulatory factor $2,4,5[I R F 2,4,5])$, adaptors, and signal transducers (TNF receptor-associated factor 6 [TRAF6] and WD repeat-containing protein 6 -like protein). Some typical genes associated with nerve development and function were also found, such as fibroblast growth factor receptor (FGFR2), neurogenic locus protein delta-like protein, neurogenic locus notch-like protein 3 (NOTCH3), paired box protein Pax-6 (PAX6), AMPHIHOX4, PAX3/7, neurotrophic tyrosine kinase receptor precursor (NTKR), NOTCH1L, and NOTCH2. Notably, OSMs regulated putative target genes encoding proteins involved in vertebrate brain function and nerve disease and visual sensing in the nerve cord, including adult brain protein 239-like, huntingtin, and opsin protein.

In the muscle, OSM-predicted targets related to ubiquitination are overrepresented, including E3 ubiquitin-protein ligase family (E3 ubiquitin-protein ligase HUWE1-like, E3 ubiquitin-protein ligase CBL-like, E3 ubiquitin-protein ligase MARCH6, and E3 ubiquitin-protein ligase MURF2) and ubiquitin conjugation factor E4 B (UBE4B). In the intestine, hepatic cecum, and gill, a large number of genes involved in the innate immune system are regulated by OSMs, including HSPs, complement component, PRRs, cytokines, apoptosis-related proteins, adaptors, signal transducers, and caspases; particularly, mucin-2 (MUC2), a major mucin of the colon mucus in the vertebrates, frequently appeared to be potentially regulated targets of OSMs in the intestine and hepatic cecum. Interestingly, in the list of predicted target genes of OSMs in the hepatic cecum, we noted some insulin-related genes, such as insulin-degrading enzyme (IDE), insulin-like growth factor, and insulin-like growth factor 1 receptor (IGF1R).

\section{Validation of miRNA Expression Profiles Using qRT-PCR}

To further validate the expression of the identified miRNAs by deep sequencing, the expression patterns of eight randomly selected known miRNAs across different organs were investigated using Taqman miRNA probes. Linear correlation analyses of the fold-changes in the relative expression levels between the miRNA sequencing and qRT-PCR results showed a significant correlation for each miRNA (Pearson correlation coefficient $>0.8$, $P<0.05)$ (Figure 3D).

\section{DISCUSSION}

In this study, approximately 34 million clean reads across eight organs of $B$. belcheri were generated, which is the largest so far obtained for sRNAs of B. belcheri, allowing us to obtain adequate miRNA molecules that are contained in different organs. Additionally, the miRNA expression levels quantified by
qRT-PCR analysis of eight randomly selected known miRNAs indicated that deep sequencing is reliable, which provided a quality guarantee for downstream bioinformatics analysis. A total of 190 miRNAs (167 known) were detected in the current study. Zhou et al. (2012) performed systematic investigation of B. floridae miRNAs using a computational pipeline to predict miRNAs from throughout the genome. The authors identified 245 predicted miRNAs, which is more than the present study. The higher number of predicted miRNAs can be attributed to false positives resulting from only using a bioinformatics genome scan under a regular threshold. More importantly, miRNAs identified in the current study can indeed be expressed in organs of adult amphioxus. However, a proportion of those detected by genome-wide bioinformatics scan may not be expressed or expressed only at low levels in adult animals under normal conditions. Some examples could be those miRNAs involved in development, immunology, and response to stress. In addition, a higher proportion $(79 / 190=41.58 \%)$ of miRNAs were predicted to be expressed in an organ-specific manner. By contrast, a previously published study reported that only 47 (19.18\%) of 245 were development-specific miRNAs (Zhou et al., 2012). This could be the result of different algorithms being used to detect specific miRNAs. Alternatively, organ function formation depends on more specific miRNA than developmental processes in amphioxus.

The vast majority of the conserved miRNAs detected here have already been annotated (only novel_mir1 was a newly detected miRNA). Previous studies showed that an average TPM (used for quantification of expression level) value of the top 30 known miRNA identified in B. belcheri averaged 2,272.4. By contrast, TPM value of the top 30 novel miRNAs was only 131.1 (Zhang et al., 2017a). The conserved miRNAs present higher expression levels than $B$. belcheri-specific miRNAs, and they were detected as known miRNAs more easily, because of higher abundance of known miRNAs than that of novel miRNAs in amphioxus. Furthermore, the majority of the OSMs detected here were found to be conserved with $B$. floridae and other vertebrates. This suggests that amphioxus OSMs play a key role in organ function formation in amphioxus speciation and contributing to the complexity of the vertebrate body. Interestingly, conserved functions of the miR-1/ miR-133 cluster in chordate muscle differentiation have been proposed, and their specific location was preliminarily assessed by in situ hybridization using amphioxus embryos (early, early-mid, and late neurulae) and early larvae (Campo-Paysaa et al., 2011). In the current study, muscle-specific expression of all miR-1 and miR-133 OSMs (e.g., bbe-miR-133-5p, bbe-miR-133-3p and bbemiR-1-5p) was observed. These observations lend additional support to the hypothesized role of miR-1 and miR-133 in amphioxus muscle differentiation. Moreover, in situ hybridization of miR-1 and miR-133 produced spatial expression data similar to that obtained through miRNA sequencing. This demonstrated the reliability of the analyses conducted in this study. The conserved novel_mir1 identified in this study is homologous to bfl-miR-4871-3p. However, the function of the miR-4871 family remains elusive. Analysis of predicted target genes showed that this conserved OSM regulates genes encoding fibroblast growth factor receptors that promote the proliferation and differentiation 
of various cells via binding cell surface-expressed receptor tyrosine kinases (Han et al., 2017). Therefore, the novel_mir1 may contribute to skin function formation in amphioxus, although further research is needed for confirmation.

The data presented here show that some OSM target genes enriched in certain GO terms are limited to organ-specific functions. Those involving epidermis development and regulation of epithelial cell proliferation were detected in the skin. In the ovary, GO terms with organ-specific functions are related to gonad development. Muscle-specific GO terms in the muscle are primarily associated with muscle cell and organ development, which is consistent with data obtained using porcine and human skeletal muscle (Baccouche et al., 2014; Martini et al., 2014). Taken together, the data suggest that OSM regulatory functions in amphioxus muscle are conserved in vertebrates. For those OSMs in the intestine, their predicted target genes are also enriched to organ-specific GO terms related to the proteolysis. Proteolysis is the breakdown of proteins into shorter peptides and amino acids to aid in the digestion of food in the intestine (Aloğlu and Öner, 2011). Therefore, proteolysis would be expected to be a key intestine-specific function among various amphioxus organs. It was also observed that some hepatic cecum-specific GO terms, such as those involving organic metabolism, are functions that have also been associated with the liver in other vertebrate species (Araújo et al., 2018). A large number of nerve cord-specific GO terms involving nervous system-related functions and development were represented, indicating that the nervous system of amphioxus is unexpectedly functionally complex with diverse function, as previously described (Benito-Gutiérrez, 2006). Interestingly, some biological processes managed by the vertebrate brain are exhibited specifically, such as circadian rhythm, learning, sleep, light response, and vision. Previous publications have proposed that the anterior of the amphioxus cerebral vesicle (neurula) is homologous to the thalamus, pretectum (as part of diencephalon that is part of the forebrain in vertebrates), and midbrain of vertebrates based on the expression pattern of developmental protein-coding genes (such HOX, OTX, DLX, etc.) (Holland and Holland, 1998; Albuixech-Crespo et al., 2017). Here, the analysis supports findings that the vertebrate brain originates from part of the ancestral nerve cord based on the perspective of miRNA expression. In the gill and notochord, GO terms involving a wide range of biological functions are overrepresented. Particularly, innate immune-related GO terms, such as those involving cell apoptosis, immune system, and defense response, were widely distributed, especially in the intestine, gill, and hepatic cecum. This wide organ distribution of immune-related GO terms may be explained by the fact that these tissues are on constant contact with seawater, which is the source of most microbial exposures.

The bbe-miR-182 family was detected as known OSMs in the amphioxus skin. As reported in previous studies, miR-182 expressed at specific levels in the skin is associated with breast cancer and basal cell carcinoma (Sand et al., 2012; Wang et al., 2013), suggesting that miR-182 is an ancient miRNA family of skin-specific regulators. Furthermore, it was predicted that a miR182 may be a key regulator of innate immune- and calmodulinrelated genes; thus, miR-182 may be a potential molecular indicator of immune system activities in the research of skin immunity of amphioxus. At the time of publication, no reports on the function of miR-2068 and miR-4873 families could be found. Here, it was observed that, in the ovary, it was predicted that the OSMs miR-2068 and miR-4873 regulate PIWIL1, which belongs to the piRNA pathway (Rengaraj et al., 2014). These observations suggest potential regulatory function of miRNAs to piRNA and the ovary immune system in amphioxus. Despite that notochord-specific GO terms are not being found, some genes involved in notochord development were predicted in the target gene analysis. These OSMs may be candidates for investigation of notochord evo-devo. It was observed that not only known OSMs that potentially target genes involving vertebrate brain function, as well as development and diseases of the nerve system, were predicted, but also those that regulate typically innate immunerelated genes. For example, Liu et al. (2016) demonstrated that amphioxus LGP2, a target of bbe-miR-4928-3p as OSMs, plays an antiviral role similar to that observed in other vertebrate species by comparing expression in Branchiostoma japonicum challenged with viral mimic poly(I:C) and untreated controls. These results provide evidence that neuroimmunity is active and that miRNAs may be mediators of the neuroimmune system in early branching chordates. In the review by Candiani (2012), the roles of mir9, mir124, and mir219 in deuterostomes were possibly associated with evolution of the central nervous system (CNS). In support of the above review, nerve cord-specific expression of these three miRNA families was observed in this study. Target genes of mir9, mir124, and mir219 were predicted using bioinformatics. In addition, several known and novel miRNAs with nerve cord-specific expression were newly identified in the current investigations. These data serve as a foundational genetic resource to support future exploration of CNS evolution in chordates. In the muscle, known OSMs (e.g., bbe-miR-1-5p, bbe-miR-133-3p, bbe-miR-2058-5p, and bbe-miR-4859-3p) are primarily involved in ubiquitination. The E3 ubiquitin ligases could function in ubiquitin-mediated muscle protein turnover, promoting skeletal muscle differentiation and myofibrillogenesis (Perera et al., 2012). Furthermore, miR-133 and miR-1 were found to be muscle-specific miRNAs conserved in vertebrates (Tani et al., 2013). The data presented here show musclespecific expression of these two miRNA families, revealing their conservation in chordates, not only in higher vertebrates. Many studies have discussed the key roles of the intestine, gill, and hepatic cecum as the most important immune organs in the defense against various insults in amphioxus, including miRNA, organ structure, and protein-coding genes (Han et al., 2010). Here, OSMs involved in immune function in these three organs were identified, suggesting that the immune system may be active and regulated by miRNAs in multiple organs of amphioxus. Notably, insulin-related enzyme and growth factor (IDE, IGF1, $I G F 1 R$ ), as predicted targets of OSMs in the hepatic cecum, are well known for playing specific key roles in the pancreatic cells of vertebrates (Zhang et al., 2007; Fernández-Díaz et al., 2018). Therefore, in later branching chordates, it is reasonable to speculate that the ancestral homologous organs of vertebrate pancreas probably have functional connection to a certain extent with the hepatic cecum. 
In summary, the identification and quantification of miRNA in different organs are a first key step in the investigation of their associated functions in amphioxus. Deep sequencing was employed to detect and quantify miRNAs in eight organs. Bioinformatics analysis was used to detect OSMs based on these miRNAs. Many of the miRNAs identified here had not been previously reported. Of note, due to largely unknown and complex miRNA functions, the regulatory roles of several OSMs in this study could not be concluded. This is the first study to explore the dynamics of miRNAs in multiple organs at a genome-wide level using deep sequencing in amphioxus. Our analysis contributes to the field's understanding of the differentiation of organ function in amphioxus.

\section{DATA AVAILABILITY}

The datasets generated for this study can be found in NCBI SRA, SRR9951226-SRR9951233.

\section{ETHICS STATEMENT}

This study was carried out in accordance with the recommendations of the Guide for the Care and Use of Laboratory Invertebrate Animals. The protocol was approved by the Ethical Committee of Researches of the Nanjing University.

\section{AUTHOR CONTRIBUTIONS}

Y-JW, L-FY, and Q-LZ conceived and designed the experiments. Q-LZ, L-FY, HW, Q-HZ, and X-XW performed the experiments. Q-LZ, L-FY, HW, Q-HZ, and Y-ML analyzed the data. Q-LZ, Y-JW, and HM wrote the paper. J-YC revised the manuscript.

\section{FUNDING}

This study was supported by the foundation of Guangxi Key Laboratory of Beibu Gulf Marine Biodiversity Conservation,

\section{REFERENCES}

Albuixech-Crespo, B., López-Blanch, L., Burguera, D., Maeso, I., SánchezArrones, L., Moreno-Bravo, J. A., et al. (2017). Molecular regionalization of the developing amphioxus neural tube challenges major partitions of the vertebrate brain. PLoS Biol. 15, e2001573. doi: 10.1371/journal.pbio.2001573

Aloğlu, H. Ş., and Öner, Z. (2011). Determination of antioxidant activity of bioactive peptide fractions obtained from yogurt. J. Dairy Sci. 94, 5305. doi: 10.3168/jds.2011-4285

Araújo, B. C., Wade, N. M., De, P. M., Rodriguesfilho, J. A., Garcia, C., De, M. C., et al. (2018). Characterization of lipid metabolism genes and the influence of fatty acid supplementation in the hepatic lipid metabolism of dusky grouper (Epinephelus marginatus). Comp. Biochem. Physiol., Part A Mol. Integr. Physiol., 219-220. doi: 10.1016/j.cbpa.2018.01.018

Arner, P., and Kulyté, A. (2015). MicroRNA regulatory networks in human adipose tissue and obesity. Nat. Rev. Endocrinol. 11, 276-288. doi: 10.1038/ nrendo. 2015.25
Beibu Gulf University (no. 2019KA01 and 2019ZB09), and the Scientific Research Foundation Project of Yunnan Education Department (2019J0050), and Natural Science Foundation of Guangxi Province (no. 2016GXNSFBA380156, 2016GXNSFCA380007), and the National Natural Science Foundation of China (no. 31960286).

\section{SUPPLEMENTARY MATERIAL}

The Supplementary Material for this article can be found online at: https://www.frontiersin.org/articles/10.3389/fgene.2019.00877/ full\#supplementary-material

SUPPLEMENTARY FILE 1 | Taqman probe information of randomly selected miRNAs used in qRT-PCR analysis.

SUPPLEMENTARY FILE 2 | Summary of tags generated from sequencing of eight organs small-RNA libraries in Branchiostoma belcheri.

SUPPLEMENTARY FILE 3 | Length distribution of adult Branchiostoma belcheri small RNA (sRNA) in each of eight organs. $X$ axis is length and $Y$ axis is number of clean reads. Warmer color means shorter length of SRNA, while cooler color refer to longer.

SUPPLEMENTARY FILE 4 | The stacking histogram for annotation of small RNAs in each of eight organs. Exon and intron $<3 \%$ tags generated by the degradation of mRNAs.

SUPPLEMENTARY FILE 5 | MicroRNAs homologous with those of vertebrates and Branchiostoma floridae in adult Branchiostoma belcheri.

SUPPLEMENTARY FILE 6 | List of novel miRNA and their precursors identified newly in eight organs of adult Branchiostoma belcheri.

SUPPLEMENTARY FILE 7 | Predicted precursor structure of all the novel miRNAs identified in this study.

SUPPLEMENTARY FILE 8 | List of organ-specific miRNAs identified in each organ of adult Branchiostoma belcheri.

SUPPLEMENTARY FILE 9 | List of the GO enrichment terms of targeted genes of organ-specific miRNAs in each organ of adult Branchiostoma belcheri.

SUPPLEMENTARY FILE 10 | The predicted targeted genes of organ-specific expressed known miRNAs in each organ of adult Branchiostoma belcheri.
Baccouche, H., Maunz, M., Beck, T., Fogarassy, P., and Beyer, M. (2014). Genomewide survey of tissue-specific microRNA and transcription factor regulatory networks in 12 tissues. Sci. Rep. 4, 5150. doi: 10.1038/srep05150

Benito-Gutiérrez, E. (2006). A gene catalogue of the amphioxus nervous system. Int. J. Biol. Sci. 2, 149-160. doi: 10.7150/ijbs.2.149

Betel, D., Wilson, M., Gabow, A., Marks, D. S., and Sander, C. (2008). The microRNA.org resource: targets and expression. Nucleic Acids Res. 36 (Database issue), D149-D153. doi: 10.1093/nar/gkm995

Cabili, M. N., Trapnell, C., Goff, L., Koziol, M., Tazonvega, B., Regev, A., et al. (2011). Integrative annotation of human large intergenic noncoding RNAs reveals global properties and specific subclasses. Gene Dev. 25, 1915-1927. doi: $10.1101 / \mathrm{gad} .17446611$

Campo-Paysaa, F., Sémon, M., Cameron, R. A., Peterson, K. J., and Schubert, M. (2011). microRNA complements in deuterostomes: origin and evolution of microRNAs. Evo. Dev. 13, 15-27. doi: 10.1111/j.1525-142X.2010.00452.x

Candiani, S. (2012). Focus on miRNAs evolution: a perspective from amphioxus. Brief. Funct. Genomics 1, 107-117. doi: 10.1093/bfgp/els004 
Candiani, S., Moronti, L., Tonelli, D. D. P., Garbarino, G., and Pestarino, M. (2011). A study of neural-related microRNAs in the developing amphioxus. Evo. Dev. 2, 1-18. doi: 10.1186/2041-9139-2-15

Chen, J. Y., Huang, D. Y., and Li, C. W. (1999). An early Cambrian craniate-like chordate. Nature 402, 518-522. doi: 10.1038/990080

Conesa, A., Götz, S., García-Gómez, J. M., Terol, J., Talón, M., and Robles, M. (2005). Blast2GO: a universal tool for annotation, visualization and analysis in functional genomics research. Bioinformatics 21, 3674-3676. doi: 10.1093/ bioinformatics/bti610

Fernández-Díaz, C. M., Escobar-Curbelo, L., López-Acosta, J. F., Lobatón, C. D., Moreno, A., Sanz-Ortega, J., et al. (2018). Insulin degrading enzyme is up-regulated in pancreatic $\beta$ cells by insulin treatment. Histol. Histopathol. 33, 11997. doi: 10.14670/HH-11-997

Friedländer, M. R., Chen, W., Adamidi, C., Maaskola, J., Einspanier, R., Knespel, S., et al. (2008). Discovering microRNAs from deep sequencing data using miRDeep. Nat. Biotechnol. 26, 407-415. doi: 10.1038/nbt1394

Friedländer, M. R., Mackowiak, S. D., Li, N., Chen, W., and Rajewsky, N. (2012). miRDeep 2 accurately identifies known and hundreds of novel microRNA genes in seven animal clades. Nucleic Acids Res. 40, 37-52. doi: $10.1093 / \mathrm{nar} / \mathrm{gkr} 688$

Gao, Y., Schug, J., McKenna, L. B., Le, L. J., Kaestner, K. H., and Greenbaum, L. E. (2011). Tissue-specific regulation of mouse MicroRNA genes in endodermderived tissues. Nucleic Acids Res. 39, 454-463. doi: 10.1093/nar/gkq782

Griffiths-Jones, S. (2006). miRBase : The microRNA sequence database. Methods Mol. Biol. 342, 129-138. doi: 10.1385/1-59745-123-1:129

Han, P., Guerreronetro, H., Estienne, A., Cao, B., and Price, C. (2017). Regulation and action of early growth response 1 in bovine granulosa cells. Reproduction 154, 547-557. doi: 10.1530/REP-17-0243

Han, Y., Huang, G., Zhang, Q., Yuan, S., Liu, J., Zheng, T., et al. (2010). The primitive immune system of amphioxus provides insights into the ancestral structure of the vertebrate immune system. Dev. Comp. Immunol. 34, 791-796. doi: 10.1016/j.dci.2010.03.009

Holland, N. D., and Chen, J. (2001). Origin and early evolution of the vertebrates: new insights from advances in molecular biology, anatomy, and palaeontology. Bioessays 23, 142-151. doi: 10.1002/ 1521-1878(200102)23:2<142::AID-BIES1021>3.0.CO;2-5

Holland, L. Z., and Holland, N. D. (1998). Developmental gene expression in amphioxus: new Insights into the evolutionary origin of vertebrate brain regions, neural crest, and rostrocaudal segmentation. Am. Zool. 38, 647-658. doi: 10.1093/ icb/38.4.647

Höner zu Siederdissen, C., Bernhart, S. H., Stadler, P. F., and Hofacker, I. L. (2011). A folding algorithm for extended rna secondary structures. Bioinformatics 27, i129-i136. doi: 10.1093/bioinformatics/btr220

Huang, S., Chen, Z., Yan, X., Yu, T., Huang, G., Yan, Q., et al. (2014). Decelerated genome evolution in modern vertebrates revealed by analysis of multiple lancelet genomes. Nat. Commun. 5, 5896-5896. doi: 10.1038/ ncomms6896

Huang, Y., Ren, H., Xiong, J., Gao, X., and Sun, X. (2017). Identification and characterization of known and novel microRNAs in three tissues of Chinese giant salamander base on deep sequencing approach. Genomics 109, 258-264. doi: 10.1016/j.ygeno.2017.04.007

Jin, P., Li, S., Sun, L., Lv, C., and Ma, F. (2017). Transcriptome-wide analysis of microRNAs in Branchiostoma belcheri upon Vibrio parahemolyticus infection. Dev. Comp. Immunol. 74, 243-252. doi: 10.1016/j.dci.2017.05.002

Kent, W. J. (2002). BLAT- the BLAST-like alignment tool. Genome Res. 12, 656664. doi: 10.1101/gr.229202

Koop, B. F., Minkley, D. R., Sutherland Ben, J. G., and Jantzen, S. G. (2011). GO Trimming: Systematically reducing redundancy in large Gene Ontology datasets. BMC Res. Notes 4, 1-9. doi: 10.1186/1756-0500-4-267

Kruger, J., and Rehmsmeier, M. (2006). Rnahybrid: microrna target prediction easy, fast and flexible. Nucleic Acids Res. 34 (Web Server), W451-W454. doi: $10.1093 / \mathrm{nar} / \mathrm{gkl} 243$

Langmead, B., Trapnell, C., Pop, M., and Salzberg, S. L. (2009). Ultrafast and memory-efficient alignment of short DNA sequences to the human genome. Genome Biol. 10, R25. doi: 10.1186/gb-2009-10-3-r25

Li, F., Xiao, Y., Huang, F., Deng, W., Zhao, H., Shi, X., et al. (2015). Spatiotemporalspecific lncRNAs in the brain, colon, liver and lung of macaque during development. Mol. Biosyst. 11, 3253-3263. doi: 10.1039/C5MB00474H
Liao, X., Yang, L., Chen, X., and Chen, J. (2017a). Identification of microRNA expression profiles in the gill, intestine and hepatic caecum of Branchiostoma belcheri. Protein Cell 8, 302-307. doi: 10.1007/s13238-016-0365-3

Liao, X., Yang, L., Zhang, Q., and Chen, J. (2017b). microRNA expression changes after lipopolysaccharide treatment in gills of amphioxus Branchiostoma belcheri. Dev. Comp. Immunol. 70, 39-44. doi: 10.1016/j.dci.2017.01.007

Liu, S., Zhang, S., and Li, L. (2009). A transferrin-like homolog in amphioxus Branchiostoma belcheri: Identification, expression and functional characterization. Mol. Immunol. 46, 3117-3124. doi: 10.1016/j.molimm. 2009.06.001

Liu, S., Liu, Y., Yang, S., Huang, Y., Qin, Q., and Zhang, S. (2016). Evolutionary conservation of molecular structure and antiviral function of a viral receptor, LGP2, in amphioxus Branchiostoma japonicum. Eur. J. Immunol. 45, 34043416. doi: 10.1002/eji.201545860

Livak, K. J., and Schmittgen, T. D. (2001). Analysis of relative gene expression data using real-time quantitative PCR and the 2- DDCT method. Methods 25, 402-408. doi: 10.1006/meth.2001.1262

Marlétaz, F., Firbas, P. N., Maeso, I., Tena, J. J., Bogdanovic, O., Perry, M., et al. (2018). Amphioxus functional genomics and the origins of vertebrate gene regulation. Nature 564, 64-70. doi: 10.1038/s41586-018-0734-6

Martini, P., Sales, G., Brugiolo, M., Gandaglia, A., Naso, F., Pittà, C. D., et al. (2014). Tissue-specific expression and regulatory networks of pig microRNAome. PLoS ONE 9, e89755. doi: 10.1371/journal.pone.0089755

Perera, S., Mankoo, B., and Gautel, M. (2012). Developmental regulation of MURF E3 ubiquitin ligases in skeletal muscle. J. Mus. Res Cell Motility 33, 107-122. doi: 10.1007/s10974-012-9288-7

Putnam, N. H., Butts, T., Ferrier, D. E. K., Furlong, R. F., Hellsten, U., Kawashima, T., et al. (2008). The amphioxus genome and the evolution of the chordate karyotype. Nature 453, 1064-1071. doi: 10.1038/nature06967

Rengaraj, D., Lee, S. I., Park, T. S., Lee, H. J., Kim, Y. M., Sohn, Y. A., et al. (2014). Small non-coding RNA profiling and the role of piRNA pathway genes in the protection of chicken primordial germ cells. BMC Genomics 15, 757. doi: 10.1186/1471-2164-15-757

Sand, M., Skrygan, M., Sand, D., Georgas, D., Hahn, S. A., Gambichler, T., et al. (2012). Expression of microRNAs in basal cell carcinoma. Br. J. Dermatol. 167, 847-855. doi: 10.1111/j.1365-2133.2012.11022.x

Satoh, N., Rokhsar, D., and Nishikawa, T. (2014). Chordate evolution and the three-phylum system. Proc. Biol. Sci. 281, 20141729. doi: 10.1098/ rspb.2014.1729

Tani, S., Kuraku, S., Sakamoto, H., Inoue, K., and Kusakabe, R. (2013). Developmental expression and evolution of muscle-specific microRNAs conserved in vertebrates. Evo. Dev. 15, 293-304. doi: 10.1111/ede.12039

Wang, P. Y., Gong, H. T., Bao-Feng, L. I., Chun-Lei, L. V., Wang, H. T., Zhou, H. H., et al. (2013). Higher expression of circulating miR-182 as a novel biomarker for breast cancer. Oncol. Lett. 6, 1681-1686. doi: 10.3892/ol.2013.1593

Yanai, I., Benjamin, H., Shmoish, M., Chalifa-Caspi, V., Shklar, M., Ophir, R., et al. (2005). Genome-wide midrange transcription profiles reveal expression level relationships in human tissue specification. Bioinformatics 21, 650-659. doi: 10.1093/bioinformatics/bti042

Yang, R., Zheng, T., Cai, X., Yu, Y., Yu, C., Guo, L., et al. (2013). Genomewide analyses of amphioxus microRNAs reveal an immune regulation via miR-92d targeting C3. J. Immunol. 190, 1491-1500. doi: 10.4049/ jimmunol.1200801

Yao, M. J., Chen, G., Zhao, P. P., Lu, M. H., Jian, J., Liu, M. F., et al. (2012). Transcriptome analysis of microRNAs in developing cerebral cortex of rat. BMC Genomics 13, 232. doi: 10.1186/1471-2164-13-232

Yuan, C., Wang, X., Geng, R., He, X., Qu, L., and Chen, Y. (2013). Discovery of cashmere goat (Capra hircus) micrornas in skin and hair follicles by solexa sequencing. BMC Genomics 14, 511. doi: 10.1186/1471-2164-14-511

Zhang, F., Sjöholm, Ã., and Zhang, Q. (2007). Attenuation of insulin secretion by insulin-like growth factor binding protein-1 in pancreatic beta-cells. Biochem. Biophy. Res. Commun. 362, 152-157. doi: 10.1016/j. bbrc.2007.07.160

Zhang, Q. L., Zhu, Q. H., Xie, Z. Q., Xu, B., Wang, X. Q., and Chen, J. Y. (2017b). Genome-wide gene expression analysis of amphioxus (Branchiostoma belcheri) following lipopolysaccharide challenge using strand-specific RNA-seq. RNA Biol. 14, 1799-1809. doi: 10.1080/ 15476286.2017.1367890 
Zhang, Q. L., Zhu, Q. H., Zhang, F., Xu, B., Wang, X. Q., and Chen, J. Y. (2017a). Transcriptome-wide analysis of immune-responsive microRNAs against poly (I:C) challenge in Branchiostoma belcheri by deep sequencing and bioinformatics. Oncotarget 8, 73590-73602. doi: 10.1080/15476286.2017.1367890

Zhong, L., Zhang, F., Zhai, Y., Cao, Y., Zhang, S., and Chang, Y. (2015). Identification and comparative analysis of complement C3-associated microRNAs in immune response of Apostichopus japonicus by high-throughput sequencing. Sci. Rep. 5, 17763. doi: 10.1038/srep17763

Zhou, X., Jin, P., Qin, S., Chen, L., and Ma, F. (2012). Systematic investigation of Amphioxus (Branchiostoma floridae) microRNAs. Gene 508, 110-116. doi: 10.1016/j.gene.2012.06.065
Conflict of Interest Statement: The authors declare that the research was conducted in the absence of any commercial or financial relationships that could be construed as a potential conflict of interest.

Copyright $\odot 2019$ Zhang, Wang, Zhu, Wang, Li, Chen, Morikawa, Yang and Wang. This is an open-access article distributed under the terms of the Creative Commons Attribution License (CC BY). The use, distribution or reproduction in other forums is permitted, provided the original author(s) and the copyright owner(s) are credited and that the original publication in this journal is cited, in accordance with accepted academic practice. No use, distribution or reproduction is permitted which does not comply with these terms. 